\title{
Rotational excursion of heart in massive pericardial effusion studied by phased-array echocardiography
}

\author{
HIROHIDE MATSUO, MASAYUKI MATSUMOTO, YASUHIKO HAMANAKA, \\ TATSUHIKO OHARA, SHOHICHI SENDA, MICHITOSHI INOUE, AND \\ HIROSHI ABE \\ From the First Department of Medicine, Osaka University Medical School, Fukushima-ku, Osaka, fapan
}

SUMMARY We have observed rotational excursion, comprising anteroposterior and right to left movement of the heart, and cardiac twist around the long axis in 2 patients with massive pericardial effusion, using a phased-array echocardiograph recently developed in this country.

Sagittal real-time cross-sections showed only the anteroposterior swing motion, but horizontal crosssections revealed a counterclockwise rotational excursion and cardiac twist during this rotational excursion. The rotational excursion consisted of a rapid and almost straight movement right and posteriorly in systole, and a slow arcing excursion left and anteriorly in diastole. The speed of this movement was not uniform. The heart disclosed dominant counterclockwise twisting motions in mid to late systole and in late diastole, synchronous with atrial contraction. These movements were described relative to an external system of co-ordinates. Controls, consisting of a normal healthy subject, and cases with mitral stenosis, mitral regurgitation, and hypertrophic cardiomyopathy, did not show any rotational excursion. In the latter 2 diseases, cardiac twists were observed, but they were less dominant than in cases with massive pericardial effusion.

Since early days of this technique, echocardiography has been reported as a reliable, noninvasive diagnostic tool for massive pericardial fluid (Edler, 1955; Feigenbaum et al., 1965, 1966). In a recent report on the simulated appearance of mitral valve prolapse and systolic anterior movement of the mitral valve in the presence of pericardial fluid, the existence of a rocking or rotary motion of the heart about its attachment to the great vessels in addition to an anteroposterior swing was suggested as a possible cause (Nanda et al., 1976). Such a rotary motion in pericardial fluid, however, has not yet been demonstrated directly. In this study we have therefore investigated the motion of the heart in massive pericardial fluid in greater detail than has previously been undertaken, using phased-array echocardiography.

\section{Subjects and methods}

Two cases of benign idiopathic chronic pericarditis, diagnosed through clinical histories and examination, and 4 controls were chosen from our file and studied. Patients with massive pericardial effusion:

Received for publication 30 May 1978
Case 1: A 72-year-old woman, whose cardiothoracic ratio was 87 per cent.

Case 2: A 65-year-old man, whose cardiothoracic ratio was 80 per cent.

Neither of these cases with pericardial effusion showed electrical alternans or cardiac tamponade.

Controls: These consisted of a normal healthy 28year-old man (one of our colleagues); a 38-year-old woman with mitral stenosis; a 21-year-old woman with mitral regurgitation, and a 68-year-old man with hypertrophic obstructive cardiomyopathy.

The prototype of a new commercially available electronic beam scanning echocardiograph (Hitachi EUB-10) was used and improved during this study. The frequency of ultrasound emitted was $2.3 \mathrm{MHz}$ at a pulse repetition rate of $4608 \mathrm{~Hz}$. The sector angle was $72^{\circ}$ and the frame rate was 18 per second. The hand-held transducer, consisting of 32 small elements, had a diameter of $27 \mathrm{~mm}$. The timing of Polaroid photographs of the real-time cross-sectional image of the heart during the cardiac cycle was displayed as an interruption of the electrocardiogram displayed on the cathode ray tube. In real-time observation and cine film recording, the end of the electrocardiogram represents the time of the image. Simultaneous recording of a standard echocardio- 
gram in a direction within the real-time crosssectional echocardiogram was available using a slave cathode ray tube. Cross-sectional echocardiograms were recorded on Polaroid film and $8 \mathrm{~mm}$ cine film at a frame rate of 18 per second. Frames from one representative cardiac cycle were superimposed for

(a)
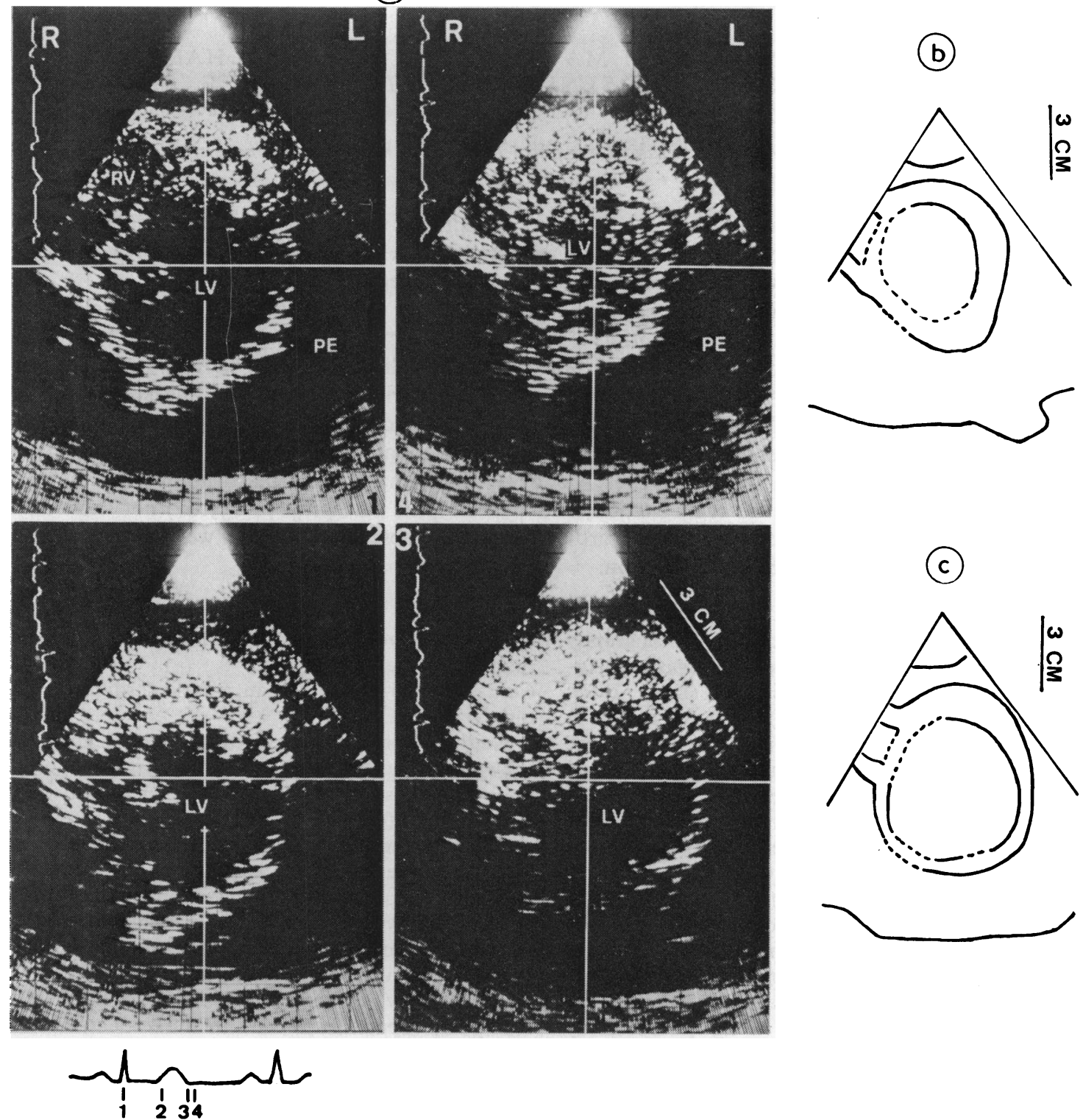

Fig. 1 (a) Rotational excursion and cardiac twist in horizontal sections at the level of the chordae tendineae of the mitral valve. These horizontal cross-sections (panels 1 to 4) were obtained at the level of the chordae tendineae of the mitral valve. Panels numbered 1 to 4 were recorded on Polaroid films at 4 different times in the cardiac phase indicated with the schematic electrocardiogram at the bottom. The heart near the anterior chest wall (panel 1) moved posteriorly and slightly to the right (panel 2) in systole, and moved slowly to the left and anteriorly showing an arching motion in diastole (panel 3, 4). Schematic representations of panels 3 and 4 for deriving the heart contours are also shown (b) and (c). Abbreviations: $R$, right; $L$, left; $P E$, pericardial effusion; $R V$, right ventricle; $L V$, left ventricle. 
the analysis of cardiac motion using an external system of co-ordinates. In order to ensure consistency and minimise experimental error in tracing, we took as reference points the point of sound source and one crossing of division lines on the cathode ray tube at constant positions in each projection of the cine frame used for tracing.

Echocardiograms were recorded while patients held their breath at expiration in a supine position. The surface of the transducer was kept in contact with the skin surface by echo transmission jelly. Cross-sectional echocardiograms were recorded in horizontal sections and in sections along the short and long axes of the heart from the anterior chest wall.

\section{Results}

(1) REAL-TIME CROSS-SECTIONAL ECHOCARDIOGRAPHIC FINDINGS IN CASE 1

(a) Horizontal cross-sections recorded at level of chordae tendineae of mitral valve

Real-time observation of case 1 in horizontal section at the level of chordae tendineae for several minutes disclosed consistently a counterclockwise rotational excursion, made up of a combination of anteroposterior and right-to-left movement, and a twisting motion of the heart as it proceeded along its path. To show this excursion 4 representative crosssectional echocardiograms recorded on Polaroid films at 4 different times in one cardiac phase are shown in Fig. 1. In this section the heart was near the anterior chest wall in early systole (Fig. 1, panel 1). As the contraction proceeded the heart showed a swift movement posteriorly and slightly to the right, taking its most posterior and medial position in mid-systole (Fig. 1, panel 2). In diastole the heart moved slowly to the left and anteriorly, showing an arcing motion (Fig. 1, panel 3), and came nearest to the anterior chest wall, nearing a position in early systole (Fig. 1, panel 4). Though the quality of the images in the Polaroid photographs as in Fig. 1 was not high, it was satisfactory for realtime observation. Rotational excursion of the heart and the magnitude of this excursion are shown in the superimposing of the contours of the whole heart (epicardium) at $\mathbf{4}$ different phases in 1 cardiac cycle (Fig. 2). These contours show a counterclockwise rotation in the course of 1 cardiac cycle.

(b) Cross-sectional echocardiograms along short axis of heart at level near apex

At a level near the apex of the heart the counterclockwise rotational excursion was more evident than at the level of chordae tendineae of the mitral

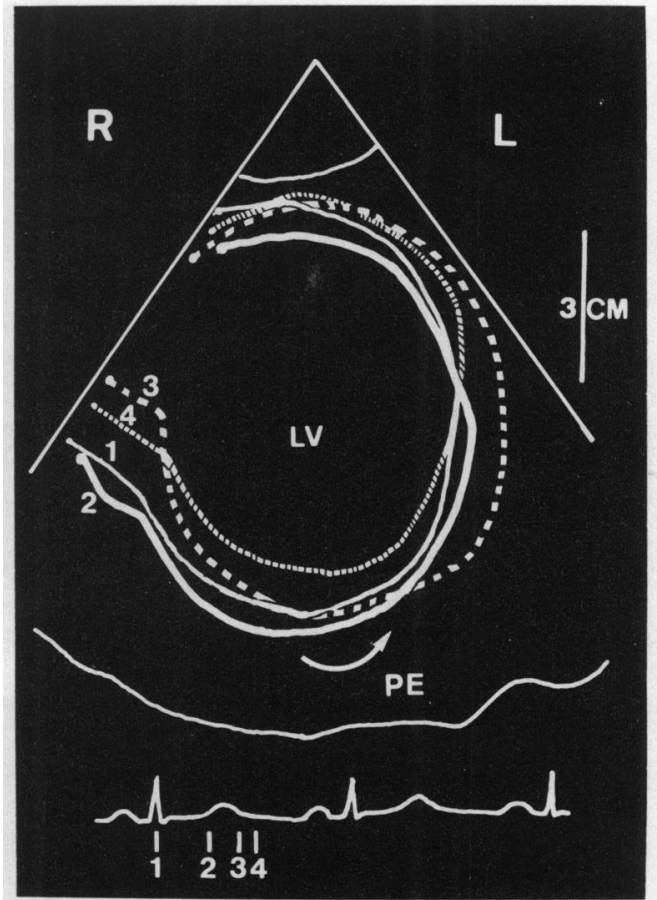

Fig. 2 Superimposed contours of the whole of the heart of Fig. 1. These contours of the whole of the heart (epicardium) showed a counterclockwise rotational excursion in a cardiac cycle. The times in the cardiac phase of these contours are indicated with the schematic electrocardiogram at the bottom of this figure.

valve in cross-sectional echocardiograms (Fig. 3). Superimposition of the contours of the whole of the heart in the 4 panels in Fig. 3 clearly shows this excursion motion of the heart (Fig. 4).

(c) Evidence of cardiac twist and rotational excursion in horizontal cross-section at level of interventricular septum

Echoes from two points at the junction of the interventricular septum and left ventricular free wall (shown in the left panel of Fig. 5a), which were identifiable in all frames of a cardiac cycle, were successively superimposed to show the additional presence of cardiac twist superimposed on rotational excursion. Representative frames used in tracing are shown in Fig. 5b. Open circles for times 1 to 8 show the changes in position in systole and solid circles for times 9 to 19 show changes in position in diastole. The timing of these points in the diagram is shown with the schematic electrocardiogram. The tracing of both points showed significant counter. clockwise rotational excursion during a cardiac cycle. The anterior point showed also counter- 

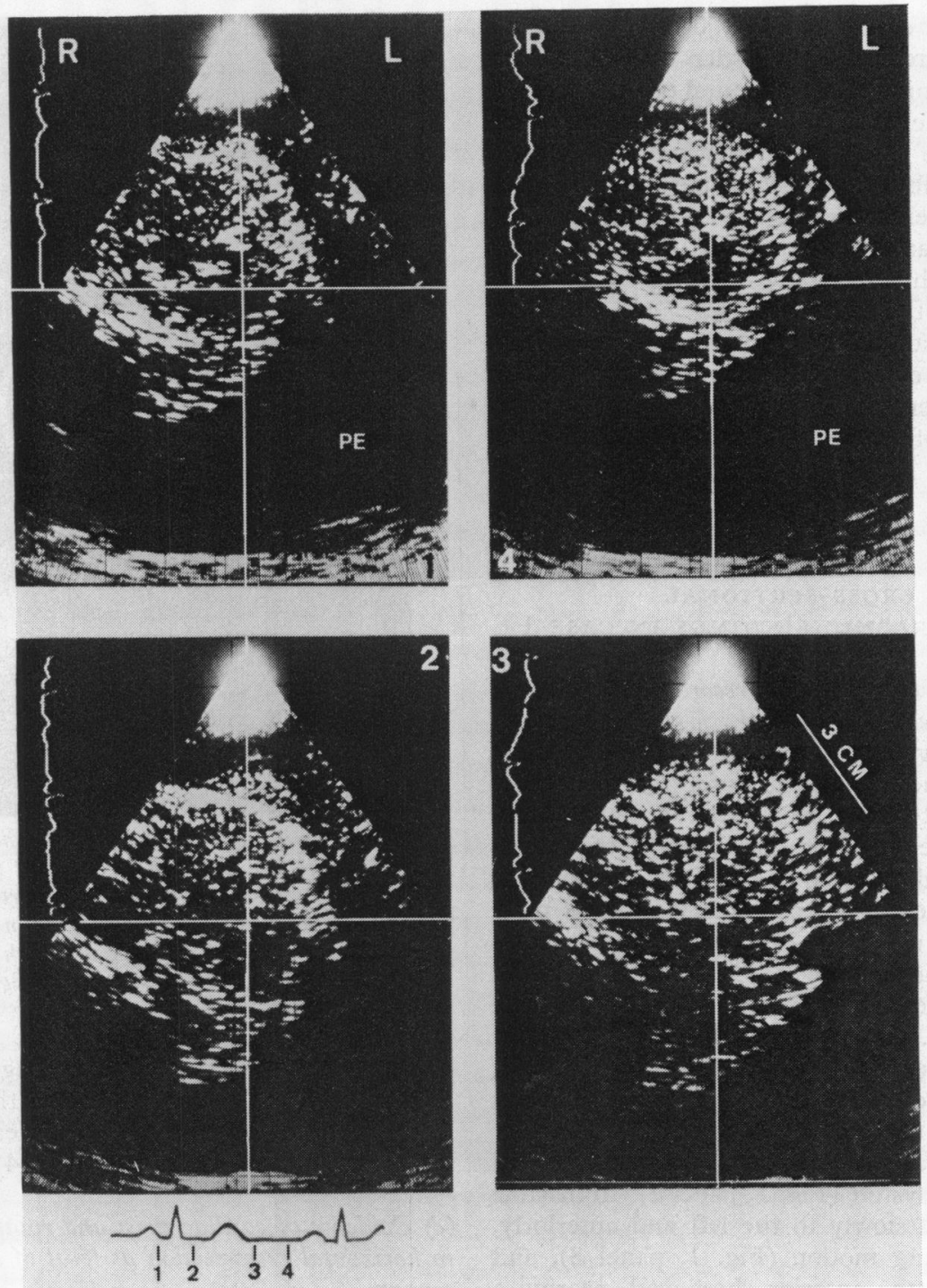

Fig. 3 Rotational excursion and cardiac twist in a section along the short axis of the heart near the apex. These cross-sections were obtained near the apex of the heart. Panels 1 to 4 were recorded on Polaroid films at the times in the cardiac phase shown with the schematic electrocardiogram. In this plane the rotational excursion showed larger excursion than at the level of the chordae tendineae of the mitral valve.

clockwise triangular motion, which corresponded to twisting motion, during times 5 to 8 in systole and during times 14 to 17 , atrial systole. The posterior point, however, did not show twisting motion.

In order to show cardiac twist, the angle of the midline of the interventricular septum to the anterior chest wall was measured successively and shown with open and solid circles in polar coordinates of Fig. 5a (right lower panel).

In this analysis dominant deflections, which indicated twist motions, were observed in mid to late systole, during times 4 to 8 , and in accordance with atrial systole during times 14 to 18 . The anterior point and midline of the interventricular 


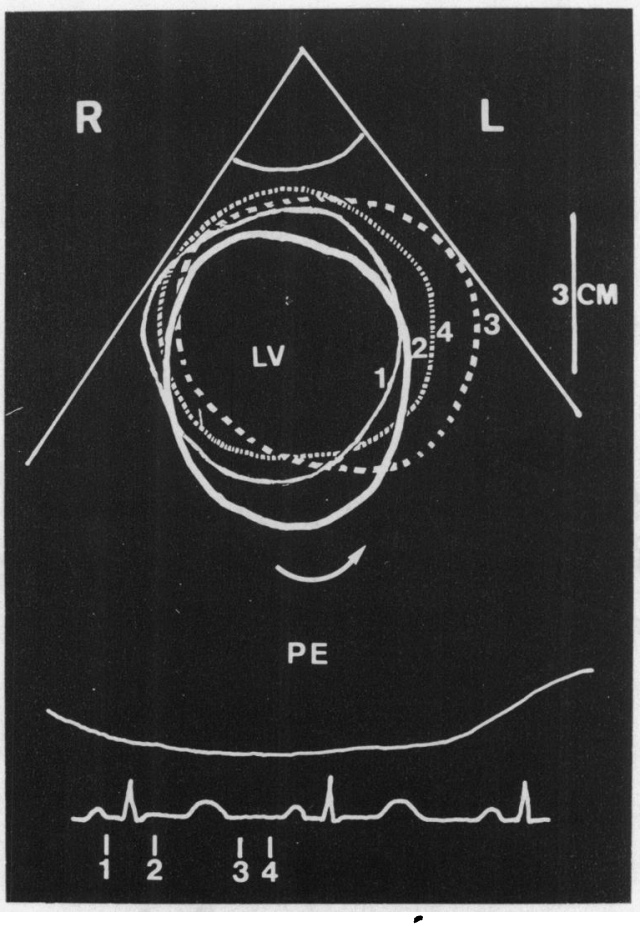

Fig. 4 Superimposed contours of the whole of the heart of Fig. 3. A large counterclockwise rotational excursion was clearly shown in this figure. The times in the cardiac phase of these contours are indicated with the schematic electrocardiogram at the bottom of this figure. The leftward shift was largest in early diastole.

septum showed similar twisting motions, but the posterior point did not show a twisting motion. The difference in motions among these may be attributed to that of their mobility resulting from anatomical differences.

(d) Cross-sectional echocardiograms along long axis of heart and standard echocardiogram within the section Anteroposterior swing motion of the heart was observed in cross-sectional echocardiograms (Fig. 6 right and centre panels), as reported previously (Matsuo et al., 1975). The heart was located posteriorly in mid-systole (centre panel), and anteriorly in diastole (right panel). The anteroposterior dimension of the left ventricle in middiastole was smaller than in end-systole in another echocardiographic cross-section along the long axis of the heart using stop action technique, suggesting the presence of lateral swing of the heart.

A standard $\mathrm{M}$-mode echocardiogram was recorded with an ultrasound beam shown as a white line in the centre and right cross-sections penetrating the anterior right ventricular wall, interventricular septum anterior mitral leaflet, and left atrioventricular groove. This echocardiogram showed a systolic posterior bowing movement on the anterior mitral leaflet, mimicking mitral valve prolapse, and a characteristic sharp posterior motion (arrow) of the epicardial surface of the left atrioventricular groove synchronous with atrial contraction. The latter seemed to correspond to the cardiac twist resulting from atrial contraction observed in the horizontal and short axis cross-sectional echocardiograms.

\section{(e) Disappearance of rotational excursion in horizontal} cross-section after diminution of pericardial fluid

A year after the initial examination by the present method a spontaneous and pronounced diminution of pericardial fluid was noted. The cardiothoracic ratio was reduced from 87 to 65 per cent. The rotational excursion of the heart was no longer seen by real-time horizontal cross-sectional echocardiography at the level of the chordae tendineae of the mitral valve. Only the cardiac twist, less dominant than before, was observed.

(2) REAL-TIME CROSS-SECTIONAL ECHOCARDIOGRAPHIC FINDINGS IN CASE 2 The rotational excursion of the heart in horizontal cross-sectional echocardiograms of this case was smaller than in case 1 (Fig. 7). Total cross-sectional echocardiographic observations in this case, including the long axis cross-sections, suggested a localisation of pericardial fluid, a lack of smoothness of rotational excursion, and a partial adhesion of the epicardium to the pericardium.

(3) REAL-TIME CROSS-SECTIONAL ECHO-

CARDIOGRAPHIC OBSERVATION IN CONTROLS

AT LEVEL OF CHORDAE TENDINEAE OF MITRAL VALVE

Four subjects (normal healthy, mitral stenosis, mitral regurgitation, hypertrophic obstructive cardiomyopathy) were chosen as controls from our file. None of these showed rotational excursion of the heart. The cardiac twist in late diastole was observed in cases with mitral regurgitation and hypertrophic obstructive cardiomyopathy, but this was less dominant than in massive pericardial effusion.

\section{Discussion}

The initial echocardiographic report on massive pericardial effusion was made by Edler in 1955. In the same year the possibility of rotatory oscillation of the heart associated with electrical alternans in massive pericardial effusion was suggested by McGregor and Baskind (1955). Feigenbaum and 


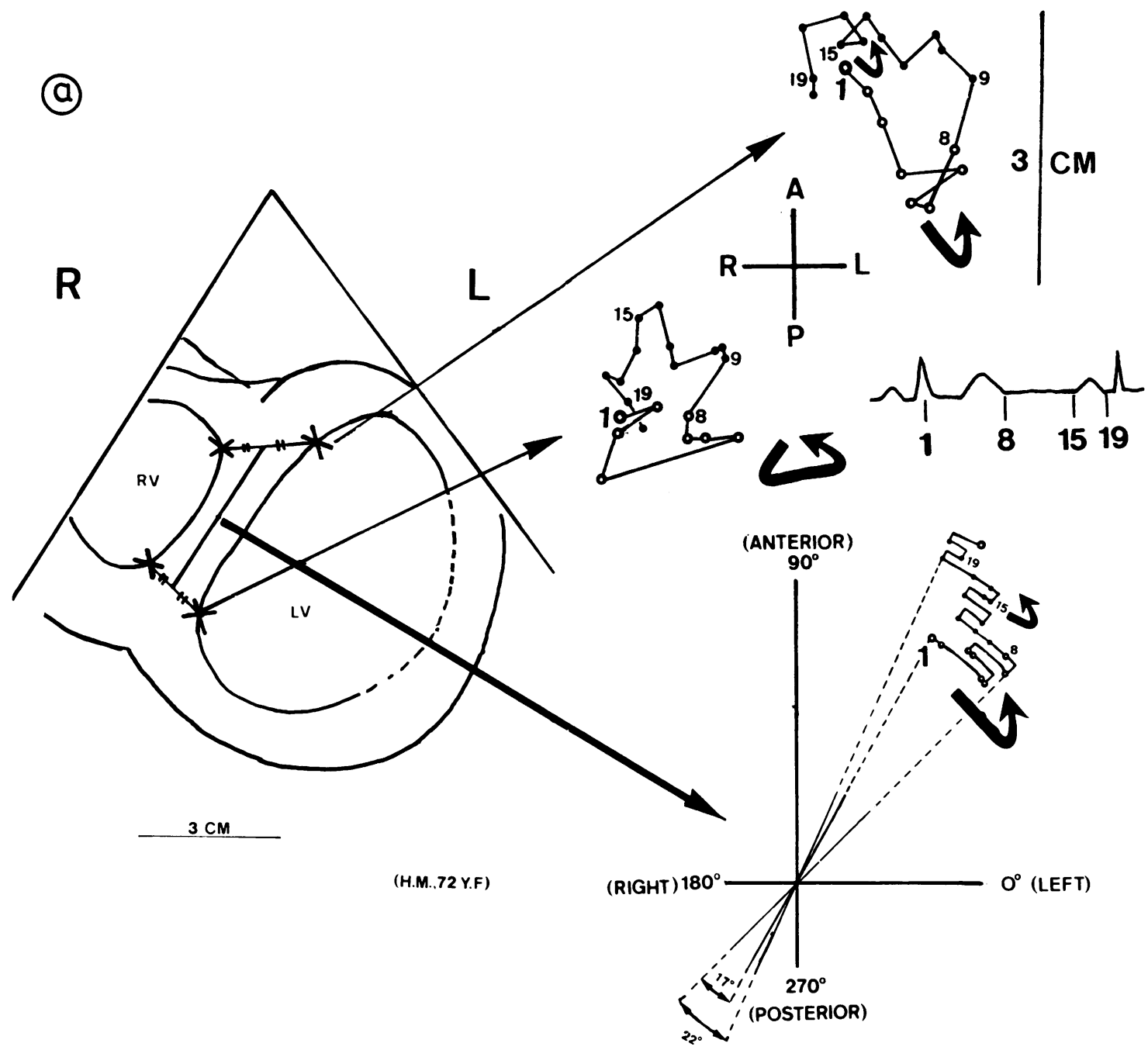

Fig. 5 Evidence of cardiac twist and rotational excursion in horizontal cross-section at the level of interventricular septum. (a) left panel: schematic representation of a horizontal cross-section indicating points and angulation of the midline of the interventricular septum for tracings. Right upper panel: tracings of 2 points at the junction of the interventricular septum and left ventricular wall as indicated on the schematic cross-section of the heart in one cardiac cycle are shown. Open circles from 1 to 8 were in systole, solid circles from 9 to 19 were in diastole. Tracings of both points showed a large rotational excursion. The anterior point showed also counterclockwise triangular motion, which corresponded to twisting motion, during times 5 to 8 in systole and a sharp counterclockwise triangular motion during 14 to 17. The posterior point did not show twisting motion. Right lower panel: The successive angulation of the interventricular septum was measured and the angles were shown in polar co-ordinates. Changes in this angle indicate cardiac twist. Cardiac twists were frequently observed throughout the cardiac cycle, but 2 dominant deflections were noticed in mid to late systole, during times 4 to 8 , and during atrial contraction, times 14 to 18. (b) Four representative frames of 20 cine frames used in tracing. The end of the electrocardiogram indicates the time of the image. AML, anterior mitral leaflet.

associates (1966) made an attempt to document this rotational movement of the heart in electrical alternans in massive pericardial effusion by standard M-mode echocardiography. However, the rotational movement was not adequately shown by this technique since only anteroposterior movement was recorded by a single ultrasonic beam fixed in one direction. In 1971 Gabor et al. reported their echo- 
(b)
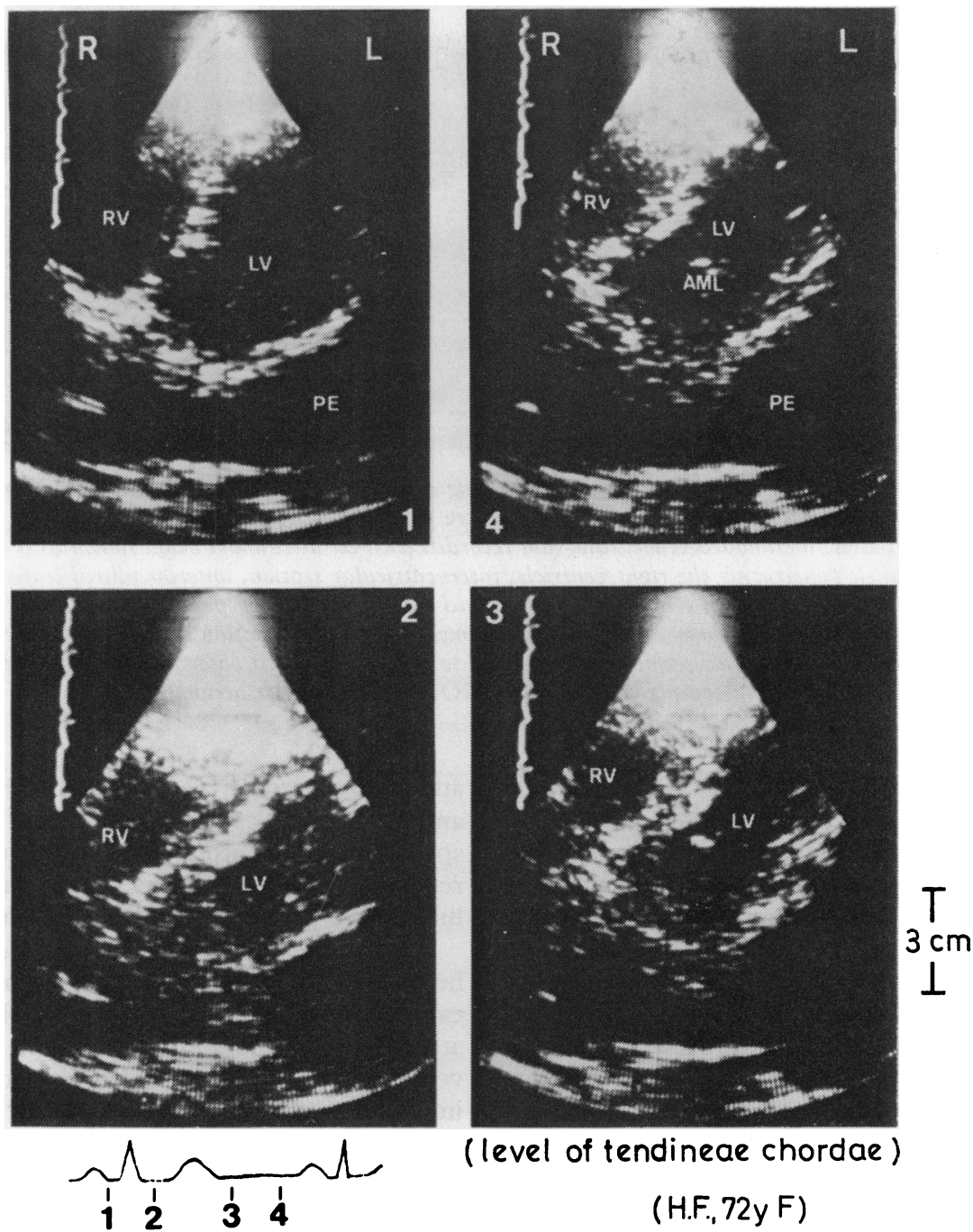

cardiographic experience in pericardial effusion in which they had to introduce lateral swing of the heart in order to understand the electrocardiogram and echocardiographic observations of their patient. They discussed electrical and mechanical alternation in pericardial effusion from the viewpoint of standard echocardiography. In 1975 Matsuo et al. also suggested the presence of lateral swing of the heart in massive pericardial effusion based on the observation that the anteroposterior dimension of the left ventricle in mid-diastole was smaller than that in end-systole in the same echocardiographic cross-section along the long axis of the heart using the stop action technique.

Real-time visualisation of the heart has now become the method of choice of documenting the rotational excursion of the heart in massive pericardial effusion. The mechanism of distortion of the M-mode echocardiogram (Feigenbaum, 1976) has also been clarified by real-time observation of the heart in horizontal cross-sections. The quality of the images in this study recorded on Polaroid films with our prototype phased array echocardiograph is not totally satisfactory, but it has been much improved by altering the behaviour of the function of a logarithmic amplifier in the receiving circuit. Real-time observation and cine films exhibit cardiac structures clearly, for they display motion which 

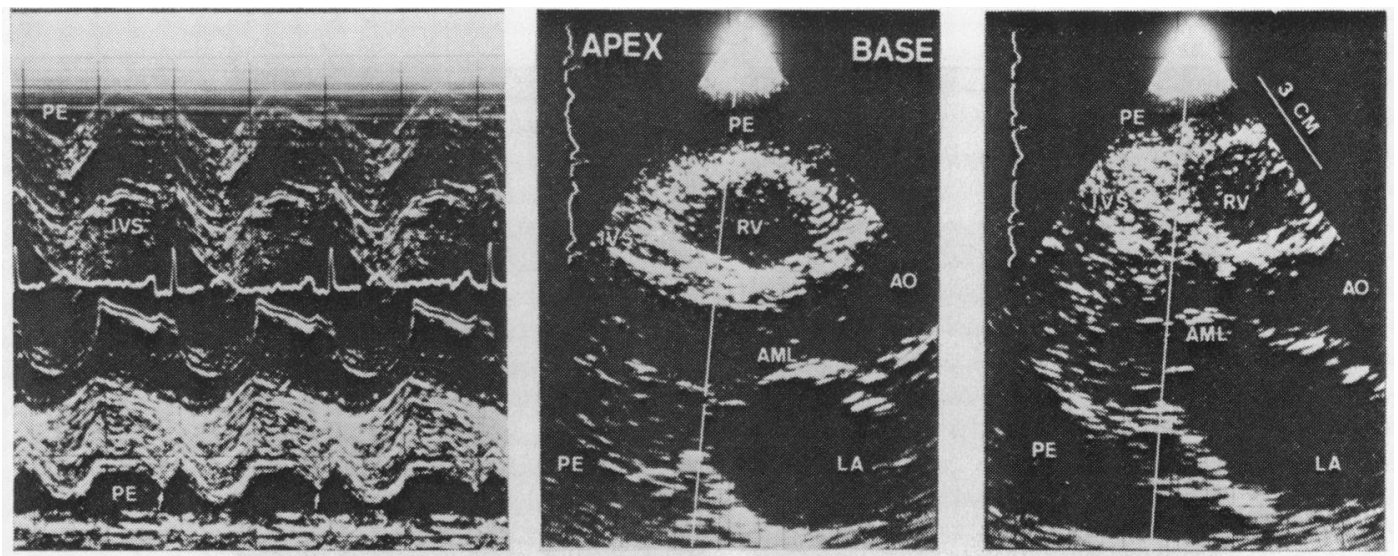

Fig. 6 Anteroposterior swing motion in sections along the long axis of the heart. Right panel: a section in middiastole revealing the heart near the anterior chest wall. Centre panel: a section in mid-systole showing a posteriorly positioned heart. Left panel: a standard echocardiogram recorded with an ultrasound beam shown as a white line in the right and centre panels penetrating the right ventricle, interventricular septum, anterior mitral leaflet, and the atrioventricular junctional portion. This echocardiogram showed a posterior bowing pattern of the anterior mitral leaflet, mimicking the mitral valve prolapse, and a sharp downward triangular motion of the epicardium at the atrioventricular junctional portion. This seemed to correspond to the cardiac twist observed in horizontal sections and caused by atrial contraction. IVS, interventricular septum; $A O$, aorta; $L A$, left atrium.

compensates for the low quality of individual images. The still Polaroid pictures exhibit an instant image which often appears poorer than real-time ones. This loss of quality is caused in part by side lobes, which lead to the appearance of ghost images and are difficult to prevent in phased-array echocardiography. Nevertheless, realtime cross-sectional echocardiography is useful for motion analysis of cardiac structures, and in the present study experimental error in tracings was avoided by maintaining two reference points at constant positions in each projection of the cine frames.

Yet to be established is the cause of this rotational excursion of the heart in massive pericardial effusion. The following factors seem responsible for this phenomenon: (i) removal of restraining influences of the pericardium together with that of the lungs and mediastinal structures on the natural rotational movement of the heart, (ii) spiral arrangement of the cardiac muscle, (iii) the asymmetry of ventricular contraction as described by McGregor and Baskind (1955) and Feigenbaum et al. (1966).

Large rotational excursion, especially in diastole, leads us to speculate that the difference in the type of blood inflow into both ventricles and/or dilatation of both ventricles may contribute to this phenomenon. The influence of heart rate (both the present cases showed slight bradycardia) must be considered. When heart rate increases to more than 100 /minute, the shortening of diastole may limit the leftward and anterior excursion, leaving only the alternative, anteroposterior pendular motion. Feigenbaum speculated that production of rotational motion requires a large, chronically accumulated, and lubricant effusion (Feigenbaum, 1976).

The counterclockwise rotational movement of the heart in congenital or acquired absence of the pericardium, to which Payvandi and Kerber (1976) attributed the paradoxical motion of the interventricular septum in pericardial defect, is also interesting, for the heart is under an increased mobility, just as in the present cases of massive pericardial effusion.

The rotational excursion of the heart in massive pericardial effusion, which we have observed, seems to occur in the presence of the above factors. Cases of massive pericardial fluid may well be seen, however, which do not have this motion because of absence of some of the above-mentioned factors, or the presence of some restraining factor, such as adhesion, which would prevent the generation of this rotational excursion.

\section{References}

Edler, I. (1955). The diagnostic use of ultrasound in heart diseases. Acta Medica Scandinavica, Suppl. 308, 32-36.

Feigenbaum, H. (1976). Echocardiography, 2nd edn., p. 428. Lea and Febiger, Philadelphia.

Feigenbaum, H., Waldhausen, J. A., and Hyde, L. P. (1965). Ultrasound diagnosis of pericardial effusion. Fournal of the American Medical Association, 191, 711-714.

Feigenbaum, H., Zaky, A., and Grabhorn, L. L. (1966). 

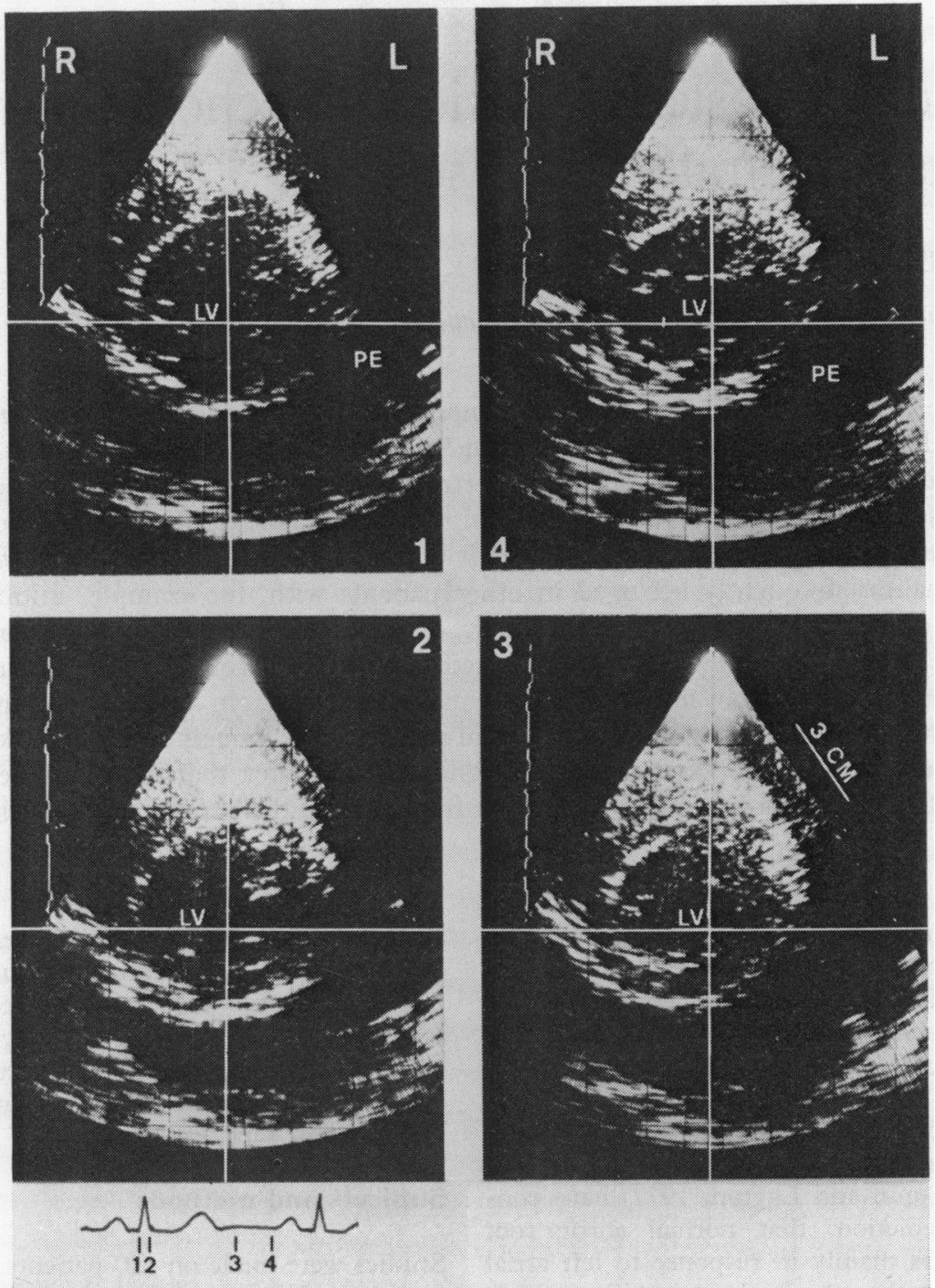

Fig. 7 Rotational excursion and cardiac twist in horizontal cross-sections at the level of chordae tendineae of the mitral valve in case 2. Massive pericardial fluid was seen in this case, but excursion of the heart was not as large as in the first case. $A$ slight localisation of the pericardial fluid was observed.

Cardiac motion in patients with pericardial effusion. A study using reflected ultrasound. Circulation, 34, 611-619.

Gabor, G. E., Winsberg, F., and Bloom, H. S. (1971). Electrical and mechanical alternation in pericardial effusion. Chest, 59, 341-344.

McGregor, M., and Baskind, E. (1955). Electric alternans in pericardial effusion. Circulation, 11, 837-843.

Matsuo, H., Kitabatake, A., Matsumoto, M., Hamanaka, Y., Beppu, S., Nagata, S., Tamai, M., Ohara, T., Senda, S., and Nimura, Y. (1975). Echocardiographic manifestation of constrictive pericarditis and pericarditis with effusion (in Japanese). Cardiovascular Sound Bulletin, 5, 173-184.

Nanda, N. C., Gramiak, R., and Gross, C. M. (1976). Echo- cardiography of cardiac valves in pericardial effusion. Circulation, 54, 500-504.

Payvandi, M. N., and Kerber, R. E. (1976). Echocardiography in congenital and acquired absence of the pericardium: an echocardiographic mimic of right ventricular volume overload. Circulation, 53, 86-92.

Requests for reprints to Dr Masayuki Matsumoto, Division of Cardiology, Department of Medicine, Albert Einstein College of Medicine, 1300 Morris Park Avenue, Bronx, NY 10461, USA. 